\title{
The LF of TP-AGB stars in the LMC/SMC
}

\author{
Gustavo Bruzual ${ }^{1}$, Stéphane Charlot ${ }^{2,3}$, Rosa González Lópezlira ${ }^{1}$, \\ Sundar Srinivasan ${ }^{4}$, Martha L. Boyer ${ }^{5}$, and David Riebel ${ }^{6}$ \\ ${ }^{1}$ Centro de Radioastronomía y Astrofísica, UNAM, Campus Morelia, México \\ email: g.bruzual@crya.unam.mx, r.gonzalez@crya.unam.mx \\ ${ }^{2}$ UPMC, UMR7095, Institut d'Astrophysique de Paris, F-75014, Paris, France \\ ${ }^{3}$ CNRS, UMR7095, Institut d'Astrophysique de Paris, F-75014, Paris, France \\ email: charlot@iap.fr \\ ${ }^{4}$ Academia Sinica, Institute of Astronomy and Astrophysics, PO Box 23-141, Taipei 10617, \\ Taiwan, R. O. C., email: sundar@asiaa.sinica.edu.tw \\ ${ }^{5}$ STScI, 3700 San Martin Drive, Baltimore, MD 21218, USA, email: mboyer@stsci.edu \\ ${ }^{6}$ Department of Physics, United States Naval Academy, 572C Holloway Road, Annapolis, MD \\ 21402, USA, email: driebel@usna.edu
}

\begin{abstract}
We show that Monte Carlo simulations of the TP-AGB stellar population in the LMC and SMC galaxies using the $\mathrm{CB}^{*}$ models produce LF and color distributions that are in closer agreement with observations than those obtained with the BC03 and CB07 models. This is a progress report of work that will be published elsewhere.
\end{abstract}

Keywords. stars: evolution, stars: AGB and post-AGB, galaxies: stellar content, galaxies: evolution

\section{Introduction}

It has been known for quite some time now that intermediate mass stars in the thermally pulsing asymptotic giant branch (TP-AGB) phase of their evolution contribute at least $50 \%$ of the NIR light in a simple stellar population (SSP) of age 1-2 Gyr, e.g., Maraston (2005), Bruzual (2007, 2011). The treatment of this stellar phase in stellar population synthesis models determines the predicted spectral energy distribution (SED) of stellar populations in this wavelength and age range. In Fig. 1 we compare the predictions of Maraston (2005) for a Salpeter IMF, $Z=Z_{\odot}$, SSP model at two different ages, with the predictions of three different versions of our code: (a) the BC03 models; (b) the minor revision of these models introduced by CB07, and (c) a major revision of this code and models (in preparation, hereafter $\mathrm{CB}^{*}$ ). The CB07 models use the same sets of stellar tracks and spectral libraries as BC03, except for the TP-AGB stars, for which CB07 follow the semi-empirical evolutionary prescriptions by Marigo \& Girardi (2007) and Marigo et al. (2008). The $\mathrm{CB}^{*}$ models used in this paper are based on the stellar evolution models computed by Bertelli et al. (2008). Tracks are available for metallicities $\mathrm{Z}=0.0001,0.0004,0.001,0.002,0.004,0.008, Z \odot=0.017,0.04$, and 0.07 . In $\mathrm{CB}^{*}$ the evolution of TP-AGB stars follows a recent prescription by Marigo \& Girardi (private communication), which has been calibrated using observations of AGB stars in the Magellanic Clouds and nearby galaxies (Girardi et al. 2010; Melbourne et al. 2012). In the optical range, the $\mathrm{CB}^{*}$ models are available for the IndoUS (Valdes et al. 2004), Miles (Sánchez-Blázquez et al. 2006), Stelib (Le Borgne et al. 2003), and BaSeL 3.1(Westera et al. 2002) spectral libraries. The NIR spectra of TP-AGB stars in CB* are selected from the compilation by Lançon \& Mouhcine (2002), the NASA Infrared Telescope Facility (IRTF) library (Rayner et al. 2009), and the C-star model atlas by 


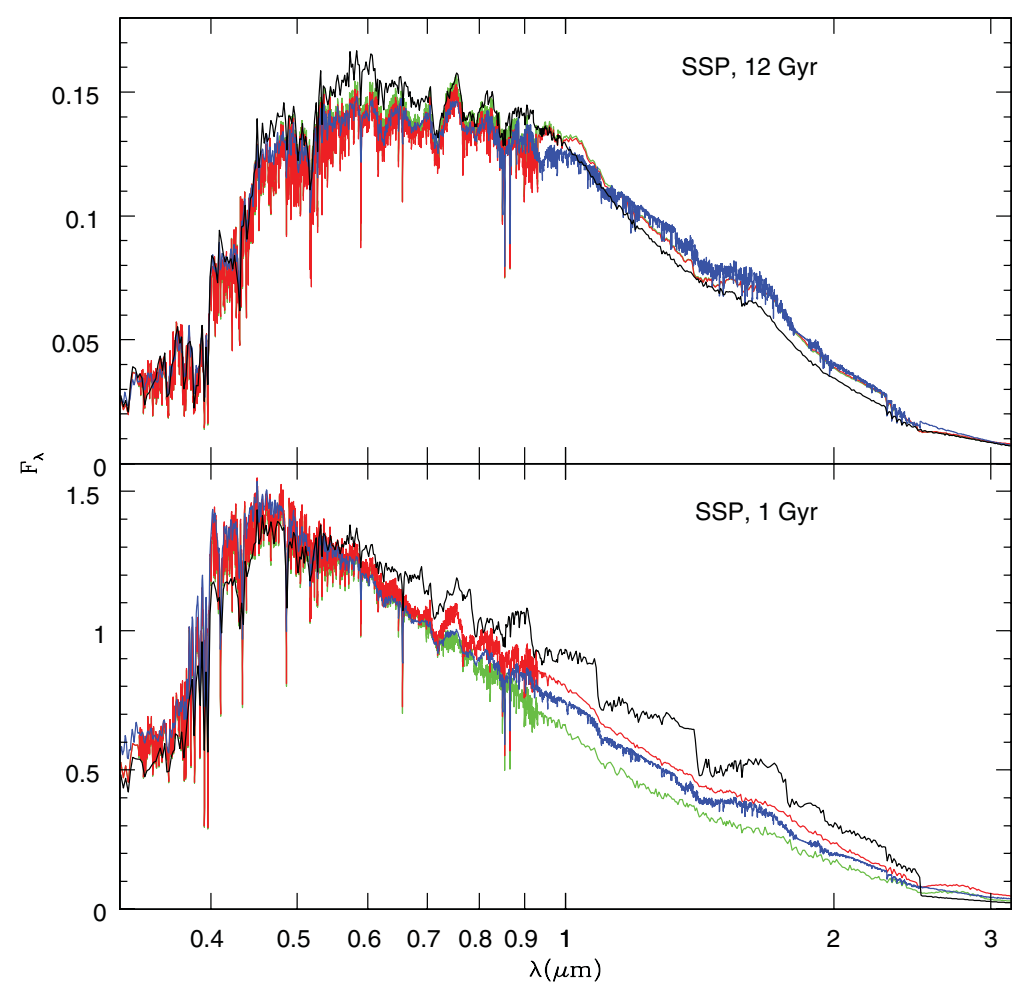

Figure 1. SED in the optical and NIR range for a Salpeter IMF, $Z=Z_{\odot}$, SSP at the age indicated inside each frame. The different lines clearly visible in the 1 Gyr frame in the 1-2 $\mu m$ range represent from bottom to top the models by $\mathrm{BC} 03, \mathrm{CB}^{*}$ (see text), CB07, and M05. Whereas the fractional range in flux spanned by these models may reach $100 \%$ at $1 \mathrm{Gyr}$, it is less than $15 \%$ at 12 Gyr. Kriek et al. (2010), Melbourne et al. (2012), and Zibetti et al. (2013) have shown evidence that the treatment of the TP-AGB stars in the CB07 and M05 models overestimates the contribution by TP-AGB stars in the NIR, favoring BC03 and CB*.

Aringer et al. (2009). The effects of mass loss and reddening in the spectra of TP-AGB stars have been included in these models as described by González-Lópezlira et al. (2010). The treatment of the TP-AGB in the M05 models is based on the Fuel Consumption Theorem and is thus completely independent of the prescriptions used in the $\mathrm{BC} / \mathrm{CB}$ models.

\section{Modeling the LF of TP-AGB stars in the LMC and SMC}

We model the distribution of TP-AGB stars in the CMD in various optical and NIR bands for a stellar population of $Z=0.008$, close to the LMC metallicity, by means of Montecarlo simulations (cf. Bruzual 2002, 2010). At each time step the mass formed in stars is derived from the LMC star formation history (Harris \& Zaritsky 2009). The stars are distributed in the CMD according to the isochrones computed with the $\mathrm{CB}^{*}$, CB07, and BC03 models described in $\S 1$. Fig. 2 shows a comparison of the LF derived from our three simulations and the observed $S A G E$ data set (Srinivasan et al. 2009) in the IRAC [4.5] $\mu \mathrm{m}$ band. The corresponding IRAC [3.6] - [4.5] $\mu \mathrm{m}$ color distributions are shown in Fig. 3. Using the same procedure and the SFH of the SMC from Harris \& Zaritsky (2004) we model the TP-AGB stellar population in the SMC galaxy. In 


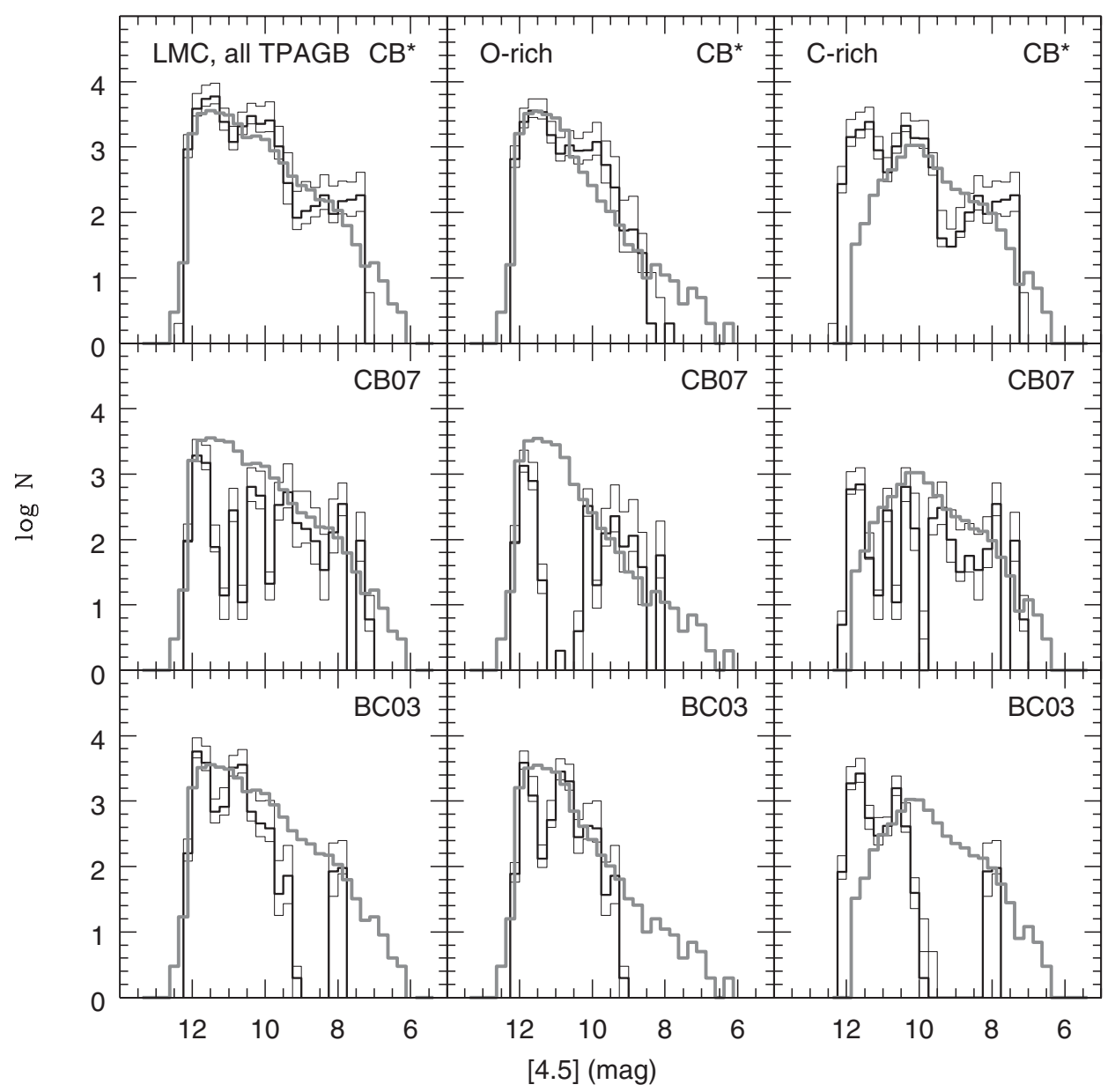

Figure 2. LF derived from our Monte Carlo simulations of the star formation history of the LMC using the $\mathrm{CB}^{*}, \mathrm{CB} 07$, and BC03 models, and from the observed $S A G E$ data set (Srinivasan et al. 2009) in the IRAC [4.5] $\mu \mathrm{m}$ band. The left column corresponds to all the TP-AGB stars. In the central and right column only the O-rich and C-rich TP-AGB stars are shown, respectively. For the simulations we assumed the Salpeter IMF, $Z=0.008$ isochrones, and we kept all the stars with apparent $K \leqslant 12 \mathrm{mag}$. The heavy gray-line corresponds to the $S A G E \mathrm{LF}$. The heavy black-line corresponds to the simulation LF using the central value of the LMC SFH (Harris \& Zaritsky 2009). The bracketing light black-lines correspond to the upper and lower limit of the $\mathrm{SFH}$ derived from the error bars given by these authors.

the case of the SMC the chemical evolution indicated by Harris \& Zaritsky (2004) is included in our simulations. Inspection of Figs. 2-4 shows that the LFs computed with the $\mathrm{CB}^{*}$ models are in closer agreement with the observations that those computed with the $\mathrm{BC} 03$ and $\mathrm{CB} 07$ models. These results are consistent with the findings by Kriek et al. (2010), Melbourne et al. (2012), and Zibetti et al. (2013) (see caption to Fig. 1), and support our choice for the treatment of TP-AGB stars in the CB* models. We do not have at hand enough information (isochrones) to perform the same kind of comparison with the M05 models. Details of this work will be published in a coming paper. 

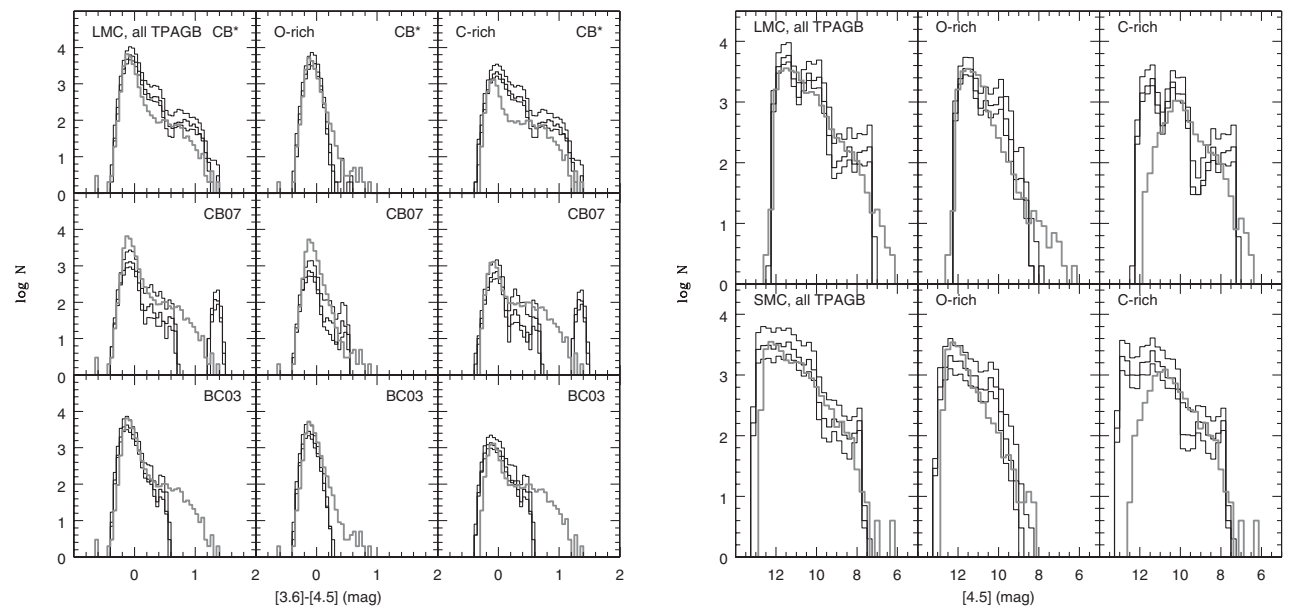

Figure 3. Same as Fig. 2 but for the IRAC [3.6] - [4.5] $\mu \mathrm{m}$ color distribution (left) and including the results of the simulations for the SMC using the SFH of Harris \& Zaritsky (2004) (right).

G. Bruzual acknowledges support from the National Autonomous University of México through grants IA102311 and IB102212.

\section{References}

Aringer, B. et al. 2009, A\&A, 503, 913

Bertelli, G. et al. 2008, A\&BA, 484, 815

Bruzual A., G. 2002, in Extragalactic Star Clusters, IAU Symposium Ser., Vol. 207, eds. D. Geisler, E. K. Grebel, \& D. Minniti, Provo:ASP, 616

Bruzual, G. 2010, RSPTA, 368, 783

Bruzual, G. 2007, in Proceedings of the IAU Symposium No. 241 "Stellar populations as building blocks of galaxies", eds. A. Vazdekis and R. Peletier, Cambridge: Cambridge University Press, 125 (arXiv:astro-ph 0703052)

Bruzual, G. 2011, in Proceedings of the XIII LARIM, eds. W. J. Henney and S. Torres-Peimbert, Rev. Mex. Astron. Astrofis., Conf. Ser., 40, 36-41

Bruzual, G. \& Charlot, S. 2003, MNRAS, 344, 1000 (BC03)

Charlot, S. \& Bruzual, G. 2007, unpublished, models distributed on demand (CB07)

Girardi, L. et al. 2010, ApJ, 724, 1030

González-Lópezlira, R. et al. 2010, MNRAS, 403, 1213

Harris, J. \& Zaritsky, D. 2004, AJ, 127,153

Harris, J. \& Zaritsky, D. 2009, AJ, 138,1243

Kriek M. et al. 2010, ApJ, 722, L64

Lançon, A. \& Mouhcine, M. 2002, A\& A, 393, 167

Le Borgne, J.-F. et al. 2003, A\&A, 402, 433

Maraston C. 2005, MNRAS, 362, 799 (M05)

Marigo, P. \& Girardi, L. 2007, A\&A, 469, 239

Marigo, P. et al. 2008, A\&SA, 482, 883

Melbourne, J. et al. 2012, ApJ, 748, 47

Rayner, J. T. et al. 2009, ApJS, 185, 289

Sánchez-Blázquez, P. et al. 2006, MNRAS, 371, 703

Srinivasan, S. et al. 2009, AJ, 137, 4810

Valdes, F. et al. 2004, ApJS, 152, 251

Westera, P. et al. 2002, A\&A 381,524

Zibetti, S. et al. 2013, MNRAS, submitted (arXiv:1205.4717) 